\section{Fenótipos corporais na adolescência: é necessário ajustar o índice de massa corporal de acordo com os estágios de maturação sexual?}

\author{
Body phenotypes in adolescence: is it necessary \\ to adjust body mass index according to sexual \\ maturation stages?
}

Fenotipos corporales en la adolescencia: ¿es necesario ajustar el índece de masa corporal

de acuerdo a las fases de madurez sexual?

\section{Resumo}

O objetivo deste trabalho é analisar o efeito da maturação sexual sobre os fenótipos corporais gerados por análise multivariada do estado nutricional de adolescentes. Este é um estudo transversal e a amostra foi composta por 833 adolescentes escolares de 10 a 15 anos, selecionados por amostragem complexa em Piracicaba, São Paulo, Brasil. Os fenótipos corporais foram definidos por análise de componentes principais (ACP), a partir da idade, dados antropométricos (massa corporal, altura, dobras cutâneas e circunferência da cintura), composição corporal (ângulo de fase medido por impedância bioelétrica) e bioquímicos (triglicerídeos, glicose, razão colesterol total/LDL, hemoglobina). Os fenótipos corporais definidos foram: $F 1_{\text {adiposidade }}$ caracterizada pela associação positiva com as variáveis dobras cutâneas, massa corporal e circunferência da cintura; $F 2_{\text {crescimento }}$ caracterizado pela associação positiva entre altura e idade; $F 3_{\text {bioquimico }}$ caracterizado pela associação positiva com razão colesterol/ LDL, triglicerídeos e glicose; e F4 metabólico caracterizado pela associação positiva com ângulo de fase, hemoglobina e negativa com glicose. Posteriormente, os fenótipos corporais foram considerados desfechos na análise dos efeitos mistos, incorporando o sexo no primeiro nível e as variáveis maturação sexual, idade, sexo e escore socioeconômico no segundo nível. Esta análise permitiu dimensionar o efeito da maturação sexual nos fenótipos corporais. Nossos resultados indicam que a maturação sexual apresentou variabilidade de explicação relevante somente sobre o fenótipo corporal que representa crescimento linear (altura e idade). Dessa forma, não é necessário ajustar as medidas antropométricas pela maturação sexual.

Adolescente; Avaliação Nutricional; Maturidade Sexual;

Composição Corporal; Biomarcadores
Jéssica Cumpian-Silva 1

Ana Elisa Madalena Rinaldi 1

Wolney Lisbôa Conde 1

doi: 10.1590/0102-311X00072719

\author{
Correspondência \\ J. Cumpian-Silva \\ Faculdade de Saúde Pública, Universidade de São Paulo. \\ Av. Dr. Arnaldo 715, São Paulo, SP 01246-904, Brasil. \\ jesscumpian@gmail.com \\ 1 Faculdade de Saúde Pública, Universidade de São Paulo, São \\ Paulo, Brasil.
}




\section{Introdução}

A avaliação do estado nutricional de adolescentes comumente é realizada com base no índice de massa corporal (IMC), sem que seja necessário classificar previamente os estágios de maturação sexual. Nessa faixa etária, a composição corporal, em especial o tecido adiposo, varia em função das idades de início e término da puberdade e da velocidade do crescimento físico ${ }^{1}$. A variabilidade na composição corporal tem potencial para alterar a associação convencional entre aumento da massa corporal, da adiposidade e risco à saúde. Esse contexto leva alguns autores a se posicionar pró ou contra o ajuste prévio da maturação sexual 1 .

Os autores que são contra o ajuste anterior pela maturação sexual argumentam que: (a) o crescimento e o desenvolvimento puberal são processos regulados principalmente por fatores biológicos e, neste sentido, a variabilidade intraestágio de maturação sexual não é intensa a ponto de modificar a classificação do estado nutricional entre os estágios de maturação sexual; (b) estima-se que diferenças inferiores a dois anos entre a média de idade da maturação sexual de uma população estudada e da população de referência não irão introduzir distorções na avaliação do estado nutricional dos indivíduos analisados 2,3,4.

Na posição oposta, os principais argumentos a favor do ajuste pela maturação sexual são: (a) o IMC varia significativamente entre os estágios da maturação sexual, destacadamente no sexo feminino; e algumas medidas antropométricas como altura, circunferência da cintura e massa corporal variam entre os estágios da maturação sexual; (b) a variabilidade do acúmulo de gordura corporal está relacionada às idades de início e término da puberdade, sua velocidade e inter-relações 5,6,7.

A possibilidade de utilizar um indicador múltiplo, físico ou latente, para avaliar o estado nutricional de adolescentes com base na ideia da multidimensionalidade tornaria mais fácil a interpretação de diferentes dimensões corporais, uma vez que permitiria analisar de forma unificada distintos parâmetros do estado nutricional (antropométrico, composição e bioquímico). Estimadores multivariados permitem o cálculo de variáveis latentes que sumarizam no mesmo vetor essa multidimensionalidade do estado nutricional. Em um estudo foi aplicada a análise de componentes principais (APC) para estimar diferentes perfis latentes do estado nutricional de adolescentes, que foram denominados fenótipos corporais. Entre os perfis criados, apenas o fenótipos corporais da adiposidade esteve fortemente associado ao IMC, já o fenótipos corporais do crescimento esteve associado ao crescimento físico linear 8 .

O objetivo do presente estudo é analisar o efeito da maturação sexual sobre os fenótipos corporais gerados por análise multivariada do estado nutricional de adolescentes.

\section{Métodos}

\section{Fonte dos dados}

Nesta análise, os dados são provenientes de adolescentes de 10 a 15 anos, residentes na cidade de Piracicaba, São Paulo, Brasil ( $\mathrm{n}=833,57 \%$ do sexo feminino). Os dados do presente estudo correspondem ao desenho transversal do inquérito intitulado Determinantes do Risco de Obesidade entre Adolescentes a partir de Inquérito de Escolares com Amostra Mista: Transversal e Longitudinal (IAP-SP). O IAP-SP corresponde à terceira onda de inquéritos realizados na cidade de Piracicaba com adolescentes escolares. Sinteticamente, seu processo amostral aconteceu por amostragem probabilística das escolas, utilizando-se dois critérios de estratificação: geográfico (centro e bairros periféricos) e tipo de administração escolar (pública e particular).

Este estudo de 2012 é, de fato, a terceira onda de estudos em Piracicaba, realizados pelo mesmo grupo de pesquisa. A primeira e segunda ondas foram realizadas em 2005 e 2009, respectivamente. Todos os estudos desenvolvidos em Piracicaba investigaram o estado de saúde e nutrição de adolescentes, em blocos de questionários distintos: comportamental, atividade física, alimentação, maturação sexual, antropometria, composição corporal, parâmetros bioquímicos e socioeconômicos. As duas primeiras ondas foram realizadas em amostra de escolas governamentais. A onda de 2009 foi realizada apenas para alunos do 5o a 9o ano do Ensino Fundamental. Em 2012, a amostra foi ampliada 
para incluir: (a) as demais séries das mesmas escolas governamentais amostradas em 2009; e (b) as escolas particulares do município. Nesse novo desenho as escolas particulares passaram a compor um novo estrato. Dessa forma, as mesmas regras probabilísticas usadas para o sorteio das escolas governamentais foram utilizadas para o sorteio das escolas particulares e, dentro dos estratos de escolas governamentais e particulares, para sortear as novas turmas. Do ponto de vista prático, a amostra de 2012 foi uma expansão das amostras de 2005 e 2009.

A coleta de dados foi realizada com a aplicação de questionários preenchidos de forma mista: demográficos e socioeconômicos foram perguntados por entrevistadores capacitados da pesquisa. Os blocos de maturação sexual e comportamento foram autopreenchidos. As medidas de massa corporal e altura foram aferidas durante a entrevista. A coleta de sangue foi realizada na própria unidade de ensino por enfermeiras habilitadas para tal procedimento.

O processo de amostragem do estudo em 2012 incluiu as mesmas 24 escolas públicas de 2009 e mais 5 escolas particulares. Todas elas foram organizadas em dois estratos geográficos denominados centro e não centro. O novo sorteio probabilístico e estruturado em múltiplos estágios obteve cobertura municipal estimada em 2.000 adolescentes escolares de ambos os sexos, matriculados em escolas públicas e privadas da cidade de Piracicaba. A amostra do estudo realizado em 2012 foi planejada como uma expansão da amostra de escolas públicas no inquérito realizado em 2009.

Para a expansão da amostra no estudo de 2012 levou-se em consideração o primeiro estágio com os anos escolares, dentro dos estratos. Dessa forma, nas redes pública e particular foram incluídos os adolescentes do 5o ao 9o ano do Ensino Fundamental e do 1o e 3o anos do Ensino Médio. As escolas do projeto de 2005 consideraram apenas do $5 \underline{\text { o a }}$ ao ano.

No segundo estágio foram consideradas as turmas alocadas em cada série. Todos os adolescentes obtiveram as mesmas chances de participar do estudo de 2012. Para a seleção dos adolescentes utilizou-se o método de amostragem sistemática, em que as escolas foram ordenadas segundo regiões (centro e não centro). Nessa etapa foram sorteadas as séries e conseguinte os alunos. Uma lista dos estudantes foi disponibilizada pela escola e a cada três escolhia-se um para participar do estudo. Para este trabalho incluímos 833 adolescentes (57\% do sexo feminino) de 10 a 15 anos avaliados em 2012.

O projeto IAP-SP foi aprovado pelo Comitê de Ética na Pesquisa da Faculdade de Saúde Pública, Universidade de São Paulo (processo no 154865, de 27 de novembro de 2012) e financiado pela Fundação de Amparo à Pesquisa do Estado de São Paulo (FAPESP), protocolo no 2009/05653-7.

\section{Variáveis antropométricas, composições corporais e bioquímicas}

A definição dos fenótipos corporais foi realizada com base nas variáveis de três dimensões mensuradas: (a) Antropometria e composição corporal: massa corporal, altura, dobras cutâneas tricipital e subescapular, circunferência da cintura e ângulo de fase. Todas as medidas antropométricas foram realizadas segundo os procedimentos recomendados pelo Manual de Referência para Padronização de Medidas Antropométricas, em duplicata e expressas pela média dos dois valores 9,10,11. O IMC foi calculado dividindo-se a massa corporal $(\mathrm{kg})$ pela altura ao quadrado $\left(\mathrm{m}^{2}\right) 12$. O ângulo de fase foi estimado por impedância bioelétrica (BIA) (modelo 0358T, RJL Systems, Clinton Township, Estados Unidos) 13,14. Os procedimentos foram realizados segundo a recomendação do manual do fabricante. O ângulo de fase foi calculado como arco tangente da razão entre reactância e resistência 15 . Todas as variáveis usadas são contínuas.

(b) Marcadores bioquímicos: Foram dosados os valores de triglicerídeos (mg/dL), glicose (glicemia de jejum $\mathrm{mg} / \mathrm{dL}$ ), dosagem de hemoglobina glicada $(\mathrm{g} / \mathrm{dL})$ e calculada a razão colesterol total/LDL (mg/dL) 15,16,17. As amostras de sangue foram coletadas após jejum de 12 horas, segundo o protocolo Técnicas para Coleta de Sangue do Ministério da Saúde 18. Todas as variáveis utilizadas são contínuas.

(c) Maturação sexual: distribuição de pelos pubianos em ambos os sexos e desenvolvimento de gônada nos meninos e mama nas meninas. Essas variáveis foram usadas como medidor de efeito em modelo linear de efeitos mistos. A referência utilizada para a avaliação da maturação sexual foi aquela proposta por Marshall \& Tanner 19,20, que está baseada nos estágios da distribuição de pelos pubianos para ambos os sexos e nos estágios do desenvolvimento das mamas em meninas ou da gônada em meninos. A avaliação foi realizada por autoclassificação, baseando-se em pranchas ilustrativas contendo as cinco fases de cada um dos critérios (pelo pubiano, gônada e mama) 21. Todos os adolescentes autoclassi- 
ficaram-se em um dos cinco estágios de maturação em cada critério. A autoclassificação é semelhante à avaliação direta por profissionais de saúde em estudos comparativos. Além disso, a Organização Mundial da Saúde (OMS) recomenda o uso da autoclassificação em estudos populacionais por diminuir custos e ser mais apropriada para superar barreiras psicossociais 22,23,24.

\section{Fenótipos corporais}

A ACP foi utilizada para definir os fenótipos corporais (variáveis latentes) com base no conjunto de medidas demográficas, antropométricas, bioquímicas e da composição corporal ${ }^{8}$. Esses fenótipos corporais foram usados como variáveis desfecho neste estudo com o objetivo de avaliar o efeito da maturação sexual sobre cada um desses fenótipos corporais.

Os componentes gerados valendo-se da ACP foram nomeados como fenótipo corporal, seguido do número que designa a ordem de variabilidade explicada e um termo que expressasse as variáveis de interesse para avaliação do estado nutricional contidas no fenótipo.

Os fenótipos corporais ( $\mathrm{F} 1$ a F4) foram gerados com base em uma proposta para que a análise do estado nutricional multivariado obtivesse os seguintes fenótipos corporais:

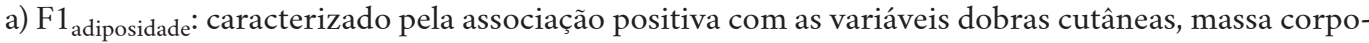
ral e circunferência da cintura.

b) $\mathrm{F} 2_{\text {crescimento }}$ : caracterizado pela associação positiva com as variáveis de altura e idade.

c) $\mathrm{F}_{\text {bioquímico }}$ : caracterizado pela associação positiva com as variáveis colesterol, triglicerídeos e glicose.

d) $\mathrm{F} 4_{\text {metabólico: }}$ caracterizado pela associação positiva com ângulo de fase, hemoglobina e associação negativa com glicose.

\section{Escore da riqueza domiciliar (escore socioeconômico)}

Para o cálculo da riqueza domiciliar foi necessário basear-se no conjunto de variáveis que descrevem os bens presentes no domicílio e na escolaridade do chefe da família. $\mathrm{O}$ escore de riqueza foi definido baseando-se na ACP como recomendado internacionalmente 25 . Esse escore pode ser usado para a predição dos escores atribuídos a cada adolescente e posteriormente dividido em quintos do escore de riqueza. As variáveis disponíveis para descrever os bens foram testadas em várias combinações, e o modelo final foi o que apresentou o maior percentual de explicação no primeiro componente da ACP e maior valor de KMO (teste Kaiser-Meyer-Olkin).

As variáveis utilizadas para formar o escore de riqueza foram as coletadas no questionário aplicado aos adolescentes na entrevista, são elas: anos de estudos do chefe da família, quantidade de cômodos da casa que as pessoas residentes utilizam para dormir, quantidade de banheiros, televisões, aparelho de ar condicionado e carros, e se possuíam bens como: rádio, geladeira ou freezer, videocassete/DVD, máquina de lavar roupa, forno de micro-ondas, telefone fixo e microcomputador. Essas variáveis de bens são dicotômicas (sim ou não).

\section{Análise dos dados}

Inicialmente, foram calculados as médias e os intervalos de 95\% de confiança (IC95\%) das variáveis idade, IMC, altura e massa corporal, segundo os estágios de maturação sexual em ambos os sexos. Foram calculados também a porcentagem e o número de adolescentes distribuídos de acordo com os estágios de maturação sexual em ambos os sexos, para as meninas: mama e pelos pubianos e para os meninos: gônada/genitália e pelos pubianos. O coeficiente de correlação de Pearson (r) foi aplicado para calcular a associação entre as variáveis antropométricas composição corporal, maturação sexual, parâmetros bioquímicos e idade em ambos os sexos.

Posteriormente, a análise do efeito da maturação sexual nos fenótipos corporais foi realizada pelo modelo de efeitos mistos com regressão linear e estimativa do coeficiente de correlação intraclasse (CCI). No nível individual foram estimados os efeitos médios, fixos, das variáveis de maturação sexual e sexo sobre os fenótipos corporais, como desfechos. Os efeitos fixos dos preditores foram expressos pelos coeficientes beta da regressão com IC95\%. 
Já no nível contextual foi estimada a variância dos efeitos dos estágios da maturação sexual. Nesse nível o modelo caracteriza-se pela estimativa de coeficientes aleatórios e estes coeficientes permitem calcular o percentual da variância entre os estágios de desenvolvimento puberal. As variáveis usadas nesse nível foram: escore socioeconômico, idade e sexo. Para expressar essa variabilidade, foi calculado o coeficiente de correlação intraclasse em cada modelo. Esses coeficientes foram utilizados para ordenar os modelos quanto à sua capacidade de explicar o estado nutricional.

A variável sexo foi alocada nos dois níveis dos modelos para estimar efeitos diferentes. No nível individual assume-se que o efeito da variável sexo é igual para todos os fenótipos corporais. Já no nível contextual, essa variável expressa a variabilidade da condição expressa pelo sexo biológico entre as fases de desenvolvimento puberal. Assim, a mesma variável pode produzir informações diferentes conforme o estimador utilizado para analisar seus efeitos sobre os estágios de Marshall \& Tanner 19,20.

A informação da idade está presente nos fenótipos corporais gerados baseando-se na ACP e também no segundo nível do modelo de efeitos mistos. Entretanto, essa informação provém de duas variáveis diferentes: a idade cronológica é representada pela idade atual do indivíduo e a idade biológica é representada pela idade do ápice do processo de maturação sexual, menarca nas meninas e espermaca nos meninos. Na ACP, a idade biológica representou a idade atual do adolescente, ou seja, os anos de vida baseados no cálculo da data de nascimento e da entrevista. No modelo de efeitos mistos, a idade cronológica representou o processo de maturação sexual, que pode ter seu início antes dos 10 anos e o término posterior aos 15, portanto, a idade cronológica é a idade de seleção da amostra. Neste caso, queríamos marcar a etapa do processo de maturação sexual e não propriamente a idade cronológica.

Foi criado um modelo linear de efeitos mistos para pelos pubianos e outro para o desenvolvimento de gônada (meninos) ou mama (meninas). Os modelos foram construídos separadamente por marcarem eixos diferentes de desenvolvimento na puberdade. Entretanto, as variáveis dos modelos foram mantidas iguais para avaliar as diferenças entre eles e compará-los.

Todas as análises foram realizadas no pacote estatístico Stata 13 (https://www.stata.com).

\section{Resultados}

A média de idade dos adolescentes deste estudo foi de 13 anos com desvio padrão (DP) de 1,2 ano. Observou-se que os valores médios de idade para ambos os sexos apresentam um aumento em todos os estágios de desenvolvimento dos pelos pubianos. As meninas apresentam um perfil diferente para o desenvolvimento de pelos pubianos segundo os indicadores: IMC, altura e massa corporal. Nos primeiros estágios de desenvolvimento puberal há um aumento dos valores médios dos indicadores citados anteriormente, e ao final nos últimos estágios de desenvolvimento de pelos pubianos há uma queda dos valores médios apresentados para as meninas. Já os meninos apresentam perfil diferenciado, segundo os estágios de desenvolvimento de pelos pubianos segundo os indicadores: IMC, altura e massa corporal há constante crescimento, com destaque para a altura (Tabela 1).

Os valores médios de idade para ambos os sexos apresentam crescimento em todos os estágios de desenvolvimento gônada e mama. Para os outros indicadores estudados (IMC, altura e massa corporal) as meninas apresentam um perfil diferente do anterior. Para as meninas existe uma tendência de crescimento nos valores médios de IMC e massa corporal. A altura também apresenta crescimento nos estágios de maturação sexual, entretanto, no último estágio há uma leve diminuição (Tabela 1).

Para os meninos o perfil apresentado também é modificado para gônada e mama. Para os indicadores IMC e massa corporal há uma tendência de diminuição dos valores médios do primeiro ao segundo estágio de maturação sexual, posteriormente, há estabilização, seguida por um aumento considerável nos indicadores. Já a altura apresenta sempre aumento dos valores médios segundo os estágios de maturação sexual (Tabela 1). Essa tendência na evolução de idade, IMC, altura e massa corporal reflete a evolução e linearidade no desenvolvimento puberal dos adolescentes em ambos os sexos. Essa tendência é significativa e reflete a evolução e a linearidade no desenvolvimento puberal dos adolescentes.

A Tabela 2 apresenta a frequência dos estágios de maturação sexual. Observamos que a maior concentração de adolescentes ocorre nos estágios 3 e 4 do desenvolvimento de pelos pubianos em 
Tabela 1

Distribuição das médias de idade, índice de massa corporal (IMC), altura e massa corporal, segundo estágio de maturação sexual em ambos os sexos. Piracicaba, São Paulo, Brasil, 2012.

\begin{tabular}{|c|c|c|c|c|c|c|c|c|}
\hline \multirow{2}{*}{$\begin{array}{l}\text { Estágios de maturação } \\
\text { sexual }\end{array}$} & \multicolumn{4}{|c|}{ Meninos (média) } & \multicolumn{4}{|c|}{ Meninas (média) } \\
\hline & $\begin{array}{l}\text { Idade } \\
\text { (anos) }\end{array}$ & $\begin{array}{c}\text { IMC } \\
\left(\mathrm{kg} / \mathrm{m}^{2}\right)\end{array}$ & $\begin{array}{l}\text { Altura } \\
(\mathrm{cm})\end{array}$ & $\begin{array}{c}\text { Massa corporal } \\
(\mathrm{kg})\end{array}$ & $\begin{array}{l}\text { Idade } \\
\text { (anos) }\end{array}$ & $\begin{array}{c}\text { IMC } \\
\left(\mathrm{kg} / \mathrm{m}^{2}\right)\end{array}$ & $\begin{array}{l}\text { Altura } \\
\text { (cm) }\end{array}$ & $\begin{array}{c}\text { Massa corporal } \\
(\mathrm{kg})\end{array}$ \\
\hline \multicolumn{9}{|l|}{ Pelo pubiano } \\
\hline 1 & 11,7 & 21,0 & 147,9 & 46,4 & 12,6 & 19,5 & 149,4 & 44,3 \\
\hline 2 & 12,0 & 20,6 & 148,2 & 46,2 & 12,4 & 21,4 & 151,4 & 50,8 \\
\hline 3 & 12,7 & 20,2 & 152,6 & 47,7 & 12,8 & 20,9 & 156,2 & 51,2 \\
\hline 4 & 13,4 & 20,6 & 161,7 & 52,1 & 13,4 & 21,7 & 158,3 & 54,5 \\
\hline 5 & 13,9 & 22,6 & 164,5 & 61,5 & 13,5 & 21,3 & 157,1 & 52,6 \\
\hline \multicolumn{9}{|l|}{ Gônada ou mama } \\
\hline 1 & 12,1 & 21,0 & 146,3 & 46,2 & 11,6 & 18,6 & 144,8 & 39,3 \\
\hline 2 & 12,0 & 20,4 & 148,8 & 46,0 & 12,0 & 19,6 & 149,5 & 44,3 \\
\hline 3 & 12,7 & 20,4 & 153,2 & 48,3 & 12,8 & 20,8 & 155,5 & 50,4 \\
\hline 4 & 13,5 & 21,2 & 162,4 & 56,3 & 13,5 & 21,9 & 158,5 & 55,7 \\
\hline 5 & 13,9 & 21,1 & 165,2 & 57,9 & 13,7 & 23,3 & 157,2 & 57,7 \\
\hline
\end{tabular}

Tabela 2

Frequência (\%) dos estágios de maturação sexual, segundo Marshall \& Tanner 19,20, em ambos os sexos. Piracicaba, São Paulo, Brasil, 2012.

\begin{tabular}{|c|c|c|c|c|c|}
\hline \multirow{3}{*}{$\begin{array}{l}\text { Estágios de maturação } \\
\text { sexual }\end{array}$} & \multicolumn{5}{|c|}{ Estágios de maturação sexual segundo Marshall \& Tanner } \\
\hline & 1 & 2 & 3 & 4 & 5 \\
\hline & $\%(n)$ & $\%(n)$ & $\%(n)$ & $\%(n)$ & $\%(n)$ \\
\hline \multicolumn{6}{|l|}{ Masculino } \\
\hline Gônada & $5,9(21)$ & $17,8(64)$ & 32,3 (116) & $33,7(121)$ & $10,0(37)$ \\
\hline Pelos pubianos & $6,1(22)$ & $19,2(69)$ & $24,0(86)$ & 38,2 (137) & $12,5(45)$ \\
\hline \multicolumn{6}{|l|}{ Feminino } \\
\hline Mama & $3,8(18)$ & $13,3(63)$ & $33,1(157)$ & $43,7(207)$ & $6,1(29)$ \\
\hline Pelos pubianos & $6,3(30)$ & $18,4(87)$ & 29,1 (139) & $34,0(162)$ & $11,6(56)$ \\
\hline
\end{tabular}

ambos os sexos; o mesmo fenômeno ocorre para o desenvolvimento de gônada nos meninos e mama nas meninas.

Nos dois sexos encontramos correlação forte e positiva entre as variáveis altura e massa corporal e as variáveis de maturação sexual (pelos pubianos, gônada e mama). Existe também correlação forte e positiva entre as variáveis antropométricas (circunferência, massa corporal e dobras cutâneas). É interessante ressaltar que as variáveis antropométricas apresentaram correlação negativa com as variáveis de maturação sexual. Outro aspecto importante é que as variáveis que representam os parâmetros bioquímicos apresentaram correlação positiva entre colesterol, triglicerídeos e glicose. Esse resultado indica um perfil de associação entre as variáveis: (a) perfil de adiposidade, associação com as variáveis de dobras cutâneas e circunferência; (b) perfil de crescimento, associação das variáveis de altura e idade; e (c) perfil bioquímico, associação das variáveis de triglicerídeos, colesterol e glicose (Tabela 3).

Nas Tabelas 4 e 5 descrevemos os resultados dos modelos de efeitos mistos. Foi criado um modelo para pelos pubianos e outro para o desenvolvimento de gônada e mama. Os modelos foram construí- 
Tabela 3

Correlação das variáveis antropométricas, composição corporal, maturação sexual, parâmetros bioquímicos e idade em ambos os sexos, feminino na parte superior e masculino na parte inferior da diagonal apresentada na tabela. Piracicaba, São Paulo, Brasil.

\begin{tabular}{|c|c|c|c|c|c|c|c|c|c|c|c|c|c|}
\hline Indicadores & Altura & Peso & $\begin{array}{l}\text { Dobra } \\
\text { cutânea } \\
\text { triciptal }\end{array}$ & $\begin{array}{c}\text { Dobra } \\
\text { cutânea } \\
\text { subesca- } \\
\text { pular }\end{array}$ & $\begin{array}{l}\text { Circunfe- } \\
\text { rência da } \\
\text { cintura }\end{array}$ & $\begin{array}{l}\text { Ângulo } \\
\text { de fase }\end{array}$ & Pelo & $\begin{array}{c}\text { Gônada/ } \\
\text { Mama }\end{array}$ & $\begin{array}{l}\text { Coles- } \\
\text { terol }\end{array}$ & $\begin{array}{l}\text { Hemo- } \\
\text { globina }\end{array}$ & $\begin{array}{l}\text { Triglice- } \\
\text { rídeos }\end{array}$ & Glicose & Idade \\
\hline Altura & 1,00 & 0,52 & 0,20 & 0,26 & 0,25 & 0,03 & 0,31 & 0,41 & 0,04 & $-0,01$ & $-0,11$ & 0,01 & 0,50 \\
\hline Massa corporal & 0,67 & 1,00 & 0,64 & 0,79 & 0,86 & 0,27 & 0,16 & 0,38 & 0,21 & $-0,01$ & 0,11 & 0,02 & 0,33 \\
\hline $\begin{array}{l}\text { Dobra cutânea } \\
\text { triciptal }\end{array}$ & 0,15 & 0,67 & 1,00 & 0,65 & 0,61 & 0,17 & 0,08 & 0,18 & 0,09 & 0,02 & 0,07 & 0,05 & 0,22 \\
\hline $\begin{array}{l}\text { Dobra cutânea } \\
\text { subescapular }\end{array}$ & 0,22 & 0,74 & 0,88 & 1,00 & 0,78 & 0,22 & 0,06 & 0,24 & 0,14 & $-0,01$ & 0,11 & $-0,03$ & 0,22 \\
\hline $\begin{array}{l}\text { Circunferência da } \\
\text { cintura }\end{array}$ & 0,39 & 0,89 & 0,81 & 0,87 & 1,00 & 0,29 & 0,07 & 0,23 & 0,26 & 0,00 & 0,20 & 0,05 & 0,17 \\
\hline Ângulo de fase & 0,21 & 0,37 & 0,23 & 0,28 & 0,37 & 1,00 & $-0,05$ & 0,12 & 0,17 & 0,07 & 0,06 & $-0,01$ & 0,12 \\
\hline Pelos & 0,52 & 0,30 & 0,01 & 0,01 & 0,11 & 0,10 & 1,00 & 0,36 & $-0,09$ & $-0,03$ & $-0,07$ & 0,08 & 0,31 \\
\hline Gônada/Mama & 0,52 & 0,25 & $-0,06$ & $-0,05$ & 0,06 & 0,10 & 0,65 & 1,00 & 0,00 & 0,05 & $-0,03$ & 0,02 & 0,45 \\
\hline Colesterol & 0,05 & 0,30 & 0,31 & 0,34 & 0,32 & 0,10 & 0,01 & $-0,03$ & 1,00 & $-0,03$ & 0,36 & $-0,13$ & $-0,03$ \\
\hline Hemoglobina & 0,13 & 0,13 & $-0,01$ & 0,07 & 0,07 & $-0,01$ & 0,02 & 0,04 & 0,13 & 1,00 & $-0,01$ & 0,02 & 0,03 \\
\hline Triglicerídeos & $-0,07$ & 0,18 & 0,24 & 0,25 & 0,28 & 0,08 & $-0,10$ & $-0,12$ & 0,38 & 0,05 & 1,00 & 0,06 & $-0,13$ \\
\hline Glicose & 0,10 & 0,09 & 0,06 & 0,07 & 0,12 & 0,01 & 0,09 & 0,19 & 0,03 & $-0,08$ & 0,15 & 1,00 & $-0,02$ \\
\hline Idade & 0,71 & 0,48 & 0,11 & 0,15 & 0,26 & 0,21 & 0,52 & 0,43 & 0,08 & 0,04 & $-0,06$ & 0,15 & 1,00 \\
\hline
\end{tabular}

\section{Tabela 4}

Coeficientes dos modelos de efeitos mistos para a distribuição de pelos pubianos, expressos nos cinco estágios de maturação, para os quatro fenótipos corporais ( $F 1_{\text {adiposidade, }} F 2_{\text {crescimento, }}$ F $_{\text {bioquímico, }} F 4_{\text {metabólico }}$ ) em adolescentes de 10 a 15 anos. Piracicaba, São Paulo, Brasil, 2012.

\begin{tabular}{|c|c|c|c|c|}
\hline & F1 adiposidade & $\mathbf{F} \mathbf{2}_{\text {crescimento }}$ & F3 bioquímico & F4 metabólico \\
\hline \multicolumn{5}{|l|}{ Efeito fixo } \\
\hline Sexo (IC95\%) & $0,2(-0,2 ; 0,7)$ & $-0,4(-0,7 ; 0,1)$ & $-0,0(-0,2 ; 0,2)$ & $-0,1(-0,3 ; 0,0)$ \\
\hline \multicolumn{5}{|l|}{ Efeito randômico } \\
\hline Sexo & $0,4(0,1 ; 1,1)$ & $0,3(0,1 ; 0,7)$ & 1,37 & 0,1 \\
\hline Idade & $0,3(0,2 ; 0,7)$ & $0,8(0,4 ; 1,5)$ & 0,0 & 1,08 \\
\hline Escore socioeconômico & $0,1(0,0 ; 0,3)$ & $0,1(0,0 ; 0,3)$ & 6,311 & 0,1 \\
\hline Probabilidade $>\chi^{2}$ & 0,0 & 0,0 & 0,9 & 0,3 \\
\hline Resíduo & 1,7 & 0,8 & 1,2 & 1,0 \\
\hline $\mathrm{CCl}(\%)$ & 31,0 & 78,0 & 0,6 & 1,1 \\
\hline
\end{tabular}

$\mathrm{CCl}$ : coeficiente de correlação intraclasse; F1 adiposidade: caracterizado pela associação positiva com as variáveis dobras

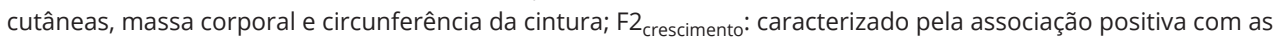

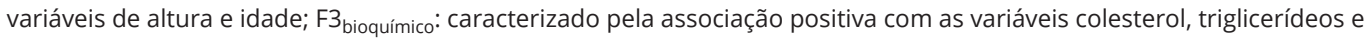
glicose; $\mathrm{F} 4$ metabólico: caracterizado pela associação positiva com ângulo de fase, hemoglobina e associação negativa com glicose; IC95\%: intervalo de 95\% de confiança.

dos separadamente por marcarem eixos diferentes de desenvolvimento na puberdade. Entretanto, as variáveis explicativas foram mantidas iguais nos dois modelos para averiguar as diferenças e semelhanças. A única diferença entre os modelos foi a mudança no segundo nível da variável pelo pubiano ou gônada e mama (resíduos). 
Tabela 5

Coeficientes do modelo de efeitos mistos para desenvolvimento de gônada e mama, expressos nos cinco estágios de maturação, para os quatro fenótipos corporais ( $F 1_{\text {adiposidade, }} \mathrm{F}_{\text {crescimento, }} \mathrm{F} 3_{\text {bioquímico, }} \mathrm{F} 4_{\text {metabólico }}$ ) em adolescentes escolares de 10 a 15 anos. Piracicaba, São Paulo, Brasil, 2012.

\begin{tabular}{|c|c|c|c|c|}
\hline & F1 adiposidade & $\mathbf{F} \mathbf{2}_{\text {crescimento }}$ & F3 bioquímico & F4 metabólico \\
\hline \multicolumn{5}{|l|}{ Efeito fixo } \\
\hline Sexo (IC95\%) & $0,3(-0,0 ; 0,7)$ & $-0,4(-0,7 ;-0,2)$ & $-0,0(-0,2 ; 0,2)$ & $-0,1(-0,3 ; 0,0)$ \\
\hline \multicolumn{5}{|l|}{ Efeito randômico } \\
\hline Sexo & 0,2 & $0,2(0,1 ; 0,6)$ & 2,08 & $0,0(2,917 ; 9,412)$ \\
\hline Idade & 1,58 & $0,8(0,4 ; 1,5)$ & 4,68 & $0,0(0,0 ; 0,14)$ \\
\hline Escore socioeconômico & 0,1 & $0,0(0,0 ; 0,2)$ & 1,49 & $0,0(0,01 ; 0,21)$ \\
\hline Probabilidade $>\chi^{2}$ & 0,0 & 0,0 & 2,0 & 0,6 \\
\hline Resíduo & 1,7 & 0,8 & 1,2 & 1,0 \\
\hline $\mathrm{CCl}(\%)$ & 2,5 & 73,0 & 0,3 & 6,9 \\
\hline
\end{tabular}

CCl: coeficiente de correlação intraclasse; F1 adiposidade: caracterizado pela associação positiva com as variáveis dobras

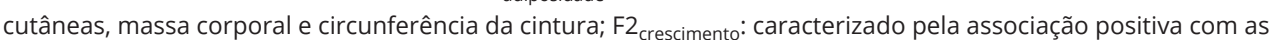
variáveis de altura e idade; F3 bioquímico: caracterizado pela associação positiva com as variáveis colesterol, triglicerídeos e glicose; F4 metabólico: caracterizado pela associação positiva com ângulo de fase, hemoglobina e associação negativa com glicose; IC95\%: intervalo de 95\% de confiança.

O F1 adiposidade apresentou variância significativa explicada para a variável sexo e $31 \%$ da variabilidade relacionada aos pelos pubianos foram explicadas neste fenótipo. Destaca-se que para o desenvolvimento de pelos pubianos existe uma considerável explicação no $\mathrm{F} 1_{\text {adiposidade, }}$ o que não acontece para gônada e mama.

No modelo de efeitos mistos construído para pelos pubianos (Tabela 4) destaca-se o resultado do $\mathrm{F} 2_{\text {crescimento }}$ que apresentou maior variância explicada pela variável alocada no nível individual (sexo). Já a variabilidade de $78 \%$ relacionada aos pelos pubianos é explicada no $\mathrm{F} 2$ crescimento.

Os fenótipos corporais 3 e 4 ( $\mathrm{F} 3_{\text {bioquímico }}$ e $\mathrm{F} 4$ metabólico $)$ apresentaram distribuição e associação inversa com a variável sexo, além disto, a variância atribuída ao desenvolvimento de pelos pubianos aproximou-se de 1\%. Portanto, não há explicação e nem efeito da maturação sexual nos fenótipos relacionados aos marcadores bioquímicos.

No modelo de efeitos mistos para análise da maturação sexual descrita segundo gônada ou mama (Tabela 5) observamos que ocorre o mesmo fenômeno visto no modelo anterior. A maior variabilidade de explicação da maturação sexual entre os fenótipos corporais está no $\mathrm{F} 2$ crescimento. As variáveis de maturação sexual são responsáveis pela explicação no F $2_{\text {crescimento }}$ de $73 \%$ da variabilidade. Para os demais fenótipos corporais a explicação atinge no máximo 0,6\%. A variância explicada pelo sexo para efeito fixo se mostrou igual ao modelo apresentado anteriormente. Um ponto importante a ser destacado é que não há explicação significante do $\mathrm{F} 1_{\text {adiposidade }}$ no modelo de gônada ou mama. Já no modelo de efeitos mistos para pelos pubianos o $\mathrm{F} 1$ adiposidade apresenta $31 \%$ de explicação. Essas evidências sugerem que o eixo metabólico expresso pelo desenvolvimento dos pelos pubianos deva estar associado ao acúmulo de adiposidade no corpo (Tabelas 4 e 5).

Os pontos fortes a serem destacados nas Tabelas 4 e 5 são: (a) a alta porcentagem de explicação atribuída à maturação sexual no $\mathrm{F} 2$ crescimento, sugerindo que esta maturação (pelos pubianos, mama ou gônada) apresenta forte correlação com este fenótipo de crescimento linear; (b) a baixa explicação das variáveis de maturação sexual (pelos pubianos, mama ou gônada) nos demais fenótipos corporais, sugerindo que os fenótipos de adiposidade e marcadores bioquímicos não são explicados pela maturação sexual (pelos pubianos, mama ou gônada). Esses resultados evidenciam que a maturação sexual não exerce efeito na composição corporal, pois não se associou ao fenótipo de adiposidade e aos fenótipos que expressam marcadores bioquímicos. 
Realizamos vários ensaios analíticos para explorar várias possibilidades no modelo de efeitos mistos. Partimos da premissa de que o modelo de efeitos mistos não deve necessariamente estar organizado de forma multinível ou hierárquica. Nesse sentido, a estimativa dos efeitos fixos ou aleatórios em cada variável obedece à organização lógica e associativa dos efeitos, sem que representem níveis ou hierarquia destes efeitos no interior do modelo analítico. Por exemplo, a atribuição de efeito aleatório às variáveis da maturação sexual está vinculada à noção de que estes efeitos modificam de forma agregada os efeitos fixos das demais variáveis no modelo, sem que estes efeitos possam ser considerados anteriores ou condicionantes dos efeitos fixos. Além disso, a análise de efeitos mistos é diferente da análise multinível e da análise hierárquica. $\mathrm{Na}$ análise de efeitos mistos todas as variáveis são alocadas nos primeiro e segundo níveis, entretanto, sem ter direção causal ou de efeito, desta forma, não foi necessário incluir uma variável agregada (distal) no modelo.

\section{Discussão}

Os aspectos a serem destacados neste trabalho sobre fenótipos corporais são principalmente o efeito significativo da maturação sexual sobre o fenótipo corporal que representa crescimento físico e a ausência de efeitos da maturação sexual na composição corporal e nos marcadores bioquímicos.

Com base no levantamento bibliográfico realizado, possivelmente este estudo é o primeiro a abordar a hipótese do efeito da maturação sexual sobre o estado nutricional de adolescentes expresso de forma multidimensional, por análise. Essa perspectiva de análise que apresentamos é ainda recente na área de estudo da avaliação nutricional e inédita no tipo de proposta aqui apresentada.

O perfil dos nossos resultados sugere que a análise isolada do IMC seja a indutora de alguns dos equívocos observados na avaliação do estado nutricional dos adolescentes durante a maturação sexual. Neste trabalho, foram incluídas separadamente a massa corporal e a altura. Acreditamos que um desses equívocos é a necessidade de ajuste anterior pela maturação sexual, desta forma, variações da massa corporal somente seriam interpretáveis plenamente quando no contexto dos respectivos estágios de maturação sexual, por serem uma derivada dos processos biológicos do desenvolvimento físico nessa etapa do ciclo vital 26,27,28.

$\mathrm{Na}$ literatura, os estudos que concluíram pela necessidade de ajuste anterior pela maturação sexual têm em comum o uso do IMC como desfecho de análise 5,6,7,29,30,31,32. Na forma de abordagem aqui apresentada, o primeiro passo foi tratar altura e massa corporal separadamente e com a mesma relevância. Nessa etapa da adolescência o uso de índices antropométricos como forma de ajuste das dimensões corporais induz à confusão, uma vez que a altura isoladamente é um bom indicador do estado nutricional na adolescência e o peso traduz múltiplas informações durante a etapa da maturação sexual, por refletir tanto a massa óssea decorrente do crescimento linear (altura) quanto as massas lábeis, como gordura e músculos, que se desenvolvem no mesmo período 33.

Os fenótipos corporais foram usados como variáveis latentes no modelo de efeitos mistos, considerando a maturação sexual como efeito randômico. Observamos nitidamente o efeito significativo da maturação sexual sobre o fenótipo corporal que expressa o crescimento físico e o efeito não significativo nos demais fenótipos corporais que expressam adiposidade, composição corporal e marcadores bioquímicos. Esse resultado não somente fortalece nosso argumento contrário à necessidade do ajuste da maturação sexual, mas também evidencia a distinção entre maturação sexual e adiposidade.

A maturação sexual na análise de efeitos mistos apresentou maior explicação e variabilidade no modelo de desenvolvimento de pelos pubianos para o $\mathrm{F} 2_{\text {crescimento. }}$ Assim, entendemos que a maturação sexual está associada fortemente com o fenótipo corporal de crescimento físico, e as alterações e modificações que ocorrem durante a fase da puberdade são altamente reguladas biologicamente ao longo desta idade.

A variabilidade de explicação da maturação sexual sobre a adiposidade e marcadores bioquímicos, expressos nos fenótipos $\mathrm{F} 1_{\text {adiposidade }} \mathrm{F} 3_{\text {bioquímico }}$ e $\mathrm{F} 4_{\text {metabólico, foi virtualmente nula. A ausência de }}$ associação observada entre maturação sexual e a adiposidade ou perfil metabólico sugere que estes processos sejam independentes, ainda que concomitantes, e a confusão observada vem das ferramentas de análise e não do desenvolvimento biológico nesse ciclo de vida. A identificação eficiente desses perfis diferentes requer ferramentas técnicas e clínicas ainda não disponíveis ou validadas. É 
importante destacar que o F4 metabólico apresentou associação positiva com as variáveis ângulo de fase e hemoglobina, e associação negativa com glicose. Esse fenótipo identifica marcadores metabólicos relacionados à massa magra.

Ao testar contra factualmente a dimensão do efeito da maturação sexual sobre os fenótipos corporais estimados por ACP, observamos nitidamente o efeito significativo da maturação sexual sobre o fenótipo corporal que expressa o crescimento físico e o efeito não significativo nos demais fenótipos corporais que expressam adiposidade, composição corporal e marcadores bioquímicos. Esse resultado não somente fortalece nosso argumento contrário à necessidade do ajuste da maturação sexual, mas também demonstra a distinção entre maturação sexual e adiposidade.

Com base nos achados, o desenvolvimento de um novo processo de avaliação clínica baseado em escores multivariados que integre a avaliação dos diferentes parâmetros antropométricos de fácil mensuração no âmbito clínico que permitam distinguir apropriadamente o processo biológico subjacente à variabilidade da massa corporal: crescimento ou adiposidade.

As principais limitações deste estudo foram: (a) informações autorreferidas para os dados de maturação sexual nos inquéritos realizados no Brasil, o que pode ter acarretado em omissão de informações corretas e dúvidas sobre o real estágio em que o adolescente se encontrava. As informações autorreferidas são comuns em estudos populacionais devido ao custo, facilidade na aplicação e superação de barreiras sociais, segundo indicado pela OMS 29,30,31,32; (b) cobertura da faixa etária: o questionário de maturação sexual foi aplicado somente nos adolescentes de 10-15 anos, considerando que nesta faixa etária está concentrada a maioria das modificações causadas pelo processo da puberdade 1 .

\section{Conclusão}

A variabilidade de explicação da maturação sexual sobre os fenótipos corporais de adiposidade e marcadores metabólicos não apresentou efeito significativo. Essa ausência de efeito sugere que a maturação sexual não está associada e nem se confunde com a variação da adiposidade ou dos marcadores bioquímicos durante a puberdade. A maturação sexual apresentou variabilidade de explicação relevante sobre o fenótipo corporal que representa crescimento linear (altura e idade). A possibilidade de utilização clínica desses dois fenótipos corporais (adiposidade e crescimento), em forma de escore, precisa ser suplementada em outros trabalhos.

\section{Colaboradores}

J. Cumpian-Silva contribuiu na concepção e projeto, análise e interpretação dos dados, na redação do artigo e revisão crítica relevante do conteúdo intelectual, e é responsável por todos os aspectos do trabalho na garantia da exatidão e integridade de qualquer parte da obra. A. E. M. Rinaldi e W. L. Conde contribuíram na redação do artigo, na revisão crítica relevante do conteúdo intelectual e na aprovação final da versão a ser publicada;

\section{Informações adicionais}

ORCID: Jéssica Cumpian-Silva (0000-0003-03623839); Ana Elisa Madalena Rinaldi (0000-00030154-554X); Wolney Lisbôa Conde (0000-00030493-134X).

\section{Agradecimentos}

Ao Conselho Nacional de Desenvolvimento Científico e Tecnológico (CNPq). Os dados foram coletados com o apoio da Fundação de Amparo à Pesquisa do Estado de São Paulo (FAPESP), projeto Determinantes do Risco de Obesidade entre Adolescentes a Partir de Inquérito de Escolares com Amostra Mista: Transversal e Longitudinal (sob o processo no 2012/01283-3). 


\section{Referências}

1. Cole TJ, Bellizzi MC, Flegal KM, Dietz WH. Establishing a standard definition for child overweight and obesity world wide: international survey. BMJ 2000; 320:1240.

2. Wang Y. Is obesity associated with early sexual maturation? A comparison of the association in American boys versus girls. Pediatrics 2002; 110:903-10.

3. Lohman TG, Going SB. Body composition assessment for development of an international growth standard for preadolescent and adolescent children. Food Nutr Bull 2006; 27(4 Suppl 5):S314-25.

4. Cole TJ, Lobstein T. Extended international (IOTF) body mass index cut-offs for thinness, overweight and obesity. Pediatr Obes 2012; 7:284-94.

5. Bini V, Celi F, Berioli MG, Bacosi ML, Stella P, Giglio P, et al. Body mass index in children and adolescents according to age and pubertal stage. Eur J Clin Nutr 2000; 54:214-8.

6. Wang Y, Moreno LA, Caballero B, Cole TJ. Limitations of the current World Health Organization Growth references for children and adolescents. Food Nutr Bull 2006; 27(4 Suppl 5):S175-88.

7. Barbosa KBF, Franceschini SCC, Priore SE. Influência dos estágios de maturação sexual no estado nutricional, antropometria e composição corporal de adolescentes. Rev Bras Saúde Matern Infant 2006; 6:375-82.

8. Cumpian-Silva J, Mazzeti CMS, Rinaldi AEM, Conde WL. Fenótipos corporais na adolescência e a maturação sexual. Cad Saúde Pública 2018; 34:e00057217.

9. Malina RM. Variation in body composition associated with sex and ethnicity. In: Heymsfield SB, Lohman TG, Wang Z, Going SB, editors. Human body composition. Champaign: Human Kinetics; 2005. p. 271-98.

10. Lohman TG, Going SB. Body composition assessment for development of an international growth standard for preadolescent and adolescent children. Food Nutr Bull 2006; 27(4 Suppl 5):S314-25.

11. Lohman TG, Roche AF, Martorell R. Anthropometric standardization reference manual. Champaign: Human Kinetics Books; 1988.

12. International Obesity Task Force. EU platform on diet, physical activity and health. http:// ec.europa.eu/health/ph_determinants/life_ style/nutrition/documents/iotf_en.pdf (acessado em 15/Set/2010).

13. Dittmar M. Reliability and variability of bioimpedance measures in normal adults: effects of age, gender and body mass. Am J Phys Anthropol 2003; 122:361-70.

14. Fernandes RA, Rosa CS, Buonani C, Oliveira AR, Freitas Júnior IF. The use of bioelectrical impedance to detect excess visceral and subcutaneous fat. J Pediatr 2007; 83:529-34.
15. Eickemberg M, Oliveira CC, Roriz AKC, Sampaio LR. Bioimpedância elétrica e sua aplicação em avaliação nutricional. Rev Nutr PUCCAMP 2011; 24:873-82.

16. Sociedade Brasileira de Cardiologia. I diretriz de prevenção da aterosclerose na infância e na adolescência. Arq Bras Cardiol 2005; 85 Suppl 6:3-36.

17. American Diabetes Association. Standards of medical care in diabetes. Diabetes Care 2005; 28 Suppl 1:4-36.

18. Ministério da Saúde. Técnicas para coleta de sangue. 3a Ed. Brasília: Ministério da Saúde; 2001.

19. Marshall WA, Tanner JM. Variations in pattern of pubertal changes in girls. Arch Dis Child 1969; 44:291-303.

20. Marshall WA, Tanner JM. Variations in pattern of pubertal changes in boys. Arch Dis Child 1970; 45:13-23.

21. Ministério da Saúde. Caderneta de saúde do adolescente. 2a Ed. Brasília: Ministério da Saúde; 2012. (Série F. Comunicação e Educação em Saúde).

22. World Health Organization. Nutrition in adolescence - issues and challenges for the health sector: issues in adolescent health and development. Geneva: World Health Organization; 2005.

23. Faria ER, Franceschini SCC, Peluzio MCG, Sant'Ana LFR, Priore SE. Aspectos metodológicos e éticos da avaliação da maturação sexual de adolescentes. Rev Paul Pediatr 2013; 31:398-405.

24. Martin RHC, Uezu R, Parra SA, Arena SS, Bojikian LP, Bohme MTS. Autoavaliação da maturação sexual masculina por meio da utilização de desenhos e fotos. Rev Paul Educ Fís 2001; 15:212-22.

25. Filmer D, Pritchett L. Estimating Wealth effects without expenditure data or tears: an application to education enrollments in states of India. Demography 2001; 38:115-32.

26. van Buuren $S$, van Schönbeck $Y$, van Dommelen P. Collection, collation and analysis of data in relation to reference heights and reference weights for female and male children and adolescents (0-18 years) in the EU, as well as in relation to the age of onset of puberty and the age at which different stages of puberty are reached in adolescents in the EU. EFSA Supporting Publication 2012; 9:EN-255.

27. Liem ET, van Buuren S, Sauer PJJ, Jaspers M, Stolk RP, Reijneveld SA. Growth during infancy and childhood, and adiposity at age 16 years: ages 2 to 7 years are pivotal. J Pediatr 2013; 162:287-92.e2

28. JI C-Y, Chen T-J. Secular changes in stature and body mass index for Chinese youth in sixteen major cities, 1950s - 2005. Am J Hum Biol 2008; 20:530-7. 
29. Lee JM, Kaciroti N, Appugliese D, Corwyn RF, Bradley RH, Lumeng JC. Body mass index and timing of pubertal initiation in boys. Arch Pediatr Adolesc Med 2010; 164:139-44.

30. Wendel D, Weber D, Leonard MB, Magge SN, Kelly A, Stallings VS, et al. Body composition estimation using skinfolds in children with and without health conditions affecting growth and body composition. Ann Hum Biol 2017; 44:108-20.

31. Buyken AE, Bolzenius K, Karaolis-Danckert N, Günther ALB, Kroke A. Body composition trajectories into adolescence according to age at pubertal growth spurt. Am J Hum Biol 2011; 23:216-24.
32. Buyken AE, Karaolis-Danckert N, Remer T. Association of prepubertal body composition in healthy girls and boys with the timing of early and late pubertal markers. Am J Clin Nutr 2009; 89:221-30.

33. Tinggaard J, Mieritz MG, Sørensen K, Mouritsen A, Hagen CP, Aksglaede L, et al. The physiology and timing of male puberty. Curr Opin Endocrinol Diabetes Obes 2012; 19:197-203. 


\section{Abstract}

This study aimed to analyze the effect of sexual maturation on body phenotypes, based on multivariate analysis of adolescents' nutritional status. This was a cross-sectional study with a sample of 833 adolescent schoolchildren 10 to 15 years of age, selected by complex sampling in Piracicaba, São Paulo State, Brazil. Body phenotypes were defined by principal components analysis (PCA), based on age, anthropometric data (body mass, height, skinfolds, and waist circumference), body composition (phase angle measured by bioelectrical impedance), and biochemical parameters (triglycerides, glucose, total cholesterol/LDL ratio, hemoglobin). Body phenotypes were classified as: $P 1_{\text {adiposity }}$ characterized as positive association with skinfolds, body mass, and waist circumference; $P 2_{\text {growth }}$ characterized as positive association between height and age; $P 3_{\text {biochemical }}$ characterized as positive association with total cholesterol/LDL ratio, triglycerides, and glucose; and $P 4_{\text {metabolic }}$ characterized as positive association with phase angle and hemoglobin and negative association with glucose. The phenotypes were later considered outcomes in the mixed effects analysis, incorporating sex at the first level and sexual maturation, age, sex, and socioeconomic score at the second level. This analysis allowed measuring the effect of sexual maturation on body phenotypes. Our results indicate that sexual maturation only a displayed relevant explanation of body phenotype that represents linear growth (height and age). Thus, it is not necessary to adjust anthropometric measures by sexual maturation.

Adolescent; Nutrition Assessment; Sexual Maturation; Body Composition; Biomarkers

\section{Resumen}

El objetivo de este trabajo es analizar el efecto de la madurez sexual sobre los fenotipos corporales generados por análisis multivariado del estado nutricional de adolescentes. Este es un estudio transversal, cuya la muestra estuvo compuesta por 833 adolescentes escolares de 10 a 15 años, seleccionados por muestra compleja en Piracicaba, São Paulo, Brasil. Los fenotipos corporales se definieron por análisis de componentes principales (ACP), a partir de la edad, datos antropométricos (masa corporal, altura, pliegues cutáneos y circunferencia de la cintura), composición corporal (ángulo de fase medido por impedancia bioeléctrica) y bioquímicos (triglicéridos, glucosa, razón colesterol total/LDL, hemoglobina). Los fenotipos corporales definidos fueron: $F 1_{\text {adiposidad }}$ caracterizada por la asociación positiva con las variables pliegues cutáneos, masa corporal y circunferencia de la cintura; $F 2_{\text {cre- }}$ cimiento, caracterizado por la asociación positiva entre altura y edad; $F 3_{\text {bioquimico, caracterizado por }}$ la asociación positiva con razón colesterol/LDL, triglicéridos y glucosa; y $\mathrm{F}_{\text {metabólico, }}$ caracterizado por la asociación positiva con ángulo de fase, hemoglobina y negativa con glucosa. Posteriormente, los fenotipos corporales fueron considerados resultados en el análisis de los efectos mixtos, incorporando el sexo en el primer nivel y las variables de madurez sexual, edad, sexo y marcador socioeconómico en el segundo nivel. Este análisis permitió dimensionar el efecto de la madurez sexual en los fenotipos corporales. Nuestros resultados indican que la madurez sexual presentó variabilidad de explicación relevante solamente sobre el fenotipo corporal que representa crecimiento lineal (altura y edad). De esta forma, no es necesario ajustar las medidas antropométricas por la madurez sexual.

Adolescente; Evaluación Nutricional; Maduración Sexual; Composición Corporal; Biomarcadores

Recebido em 16/Abr/2019

Versão final reapresentada em 29/Nov/2019

Aprovado em 06/Dez/2019 